\title{
On Weak Solvability of Boundary Value Problem with Variable Exponent Arising in Nanostructure
}

\author{
Hicham Maadan $\mathbb{D}^{1}{ }^{1}$ Nour Eddine Askour, ${ }^{1}$ and Jamal Messaho $\mathbb{D}^{2}$ \\ ${ }^{1}$ Laboratory of Applied Mathematics and Scientific Computing, Sultan Moulay Sliman University, P.O. Box 523, \\ Beni Mellal 23000, Morocco \\ ${ }^{2}$ CRMEF of Beni Mellal-Khénifra, Morocco
}

Correspondence should be addressed to Hicham Maadan; hicham.maadan@gmail.com

Received 12 May 2021; Accepted 29 June 2021; Published 23 July 2021

Academic Editor: Salah Mahmoud Boulaaras

Copyright (c) 2021 Hicham Maadan et al. This is an open access article distributed under the Creative Commons Attribution License, which permits unrestricted use, distribution, and reproduction in any medium, provided the original work is properly cited.

This work is devoted to study the limit behavior of weak solutions of an elliptic problem with variable exponent, in a containing structure, of an oscillating nanolayer of thickness and periodicity parameter depending on $\varepsilon$. The generalized Sobolev space is constructed, and the epiconvergence method is considered to find the limit problem with interface conditions.

\section{Introduction}

Nanotechnology is the science that deals with matter at the scale of one billionth of a meter and is also the study of manipulating matter at the atomic and molecular scale. The development of nanotechnology requires the collaboration of all of the sciences. At the nanolevel, the physical properties of materials such as magnetic, electric, elastic, and thermal properties are fundamentally different from those at the macroscale, and it is important to appreciate at the outset that all such properties depend on scale as well as on the material. A nanoparticle is the most fundamental component in the fabrication of a nanostructure. Its size spans the range between 1 and $100 \mathrm{~nm}$. Metallic nanoparticles have different physical and chemical properties from bulk metals (e.g., lower melting points, higher specific surface areas, specific optical properties, mechanical strengths, and specific magnetizations), properties that might prove attractive in various industrial applications. Nanostructures can also provide solutions to technological and environmental challenges in the areas of catalysis, biology, water treatment, energy conversion, and medicine. In biophysics, a mathematical anatomy of the cardiac left ventricle was considered by Koshelev et al. in [1], where the ventricle is composed of surfaces that model myo- cardial layers, each layer is filled with curves corresponding to myocardial fibers.

Since superconductivity transition temperature rises so that particle diameter is small (less than $1 \mathrm{~nm}$ ), it can be used to make high-temperature superconductivity material. Our aim is to study the composition of interconnected constitute parts in the nanoscale region where certain properties are amplified, and news behaviors and fundamental function of systems appear. Hence, there are three important effects which distinguish nanobehavior, and which do not occur in the macroenvironment; there are interfacial or surface effects, the effects of scale, and quantum effects due to a changed electronic structure. All three effects are interdependent and consequences of the extremely small size. The first plays a stone role in the kinetic theory of gases. In fact, nanoparticles are not inert in the host material, and that there is some interaction between the nanoparticles and the surrounding material, which is often modeled by a layer, which surrounds the nanoparticles but having different physical properties. Furthermore, to successfully develop nanotechnology, the major challenges are to properly understand material properties and also to be able to correctly predict their behavior when they interact with the system environment. Hence, we discuss mathematical models for problems of the enhanced 
thermal conductivity of nanofluids where potential mechanisms of enhanced heat transport in nanofluids are considered like high heat transport in the nanoparticles, since their thermal conductivity increases in a nonlinear way because most of their atoms are on the surface, and liquid nanolayer at the particle surface, which has a higher thermal conductivity than the liquid itself and so on, see for example [2].

To do so, let us consider a body which occupies a bounded three-dimensional domain, $\Omega \subset \mathbb{R}^{3}$, with a Lipschitz boundary $\partial \Omega$, composed on a nanolayer $B_{\varepsilon}$, with oscillating border $\Sigma_{\varepsilon}^{ \pm}$, of middle interface $\Sigma$, where $B_{\varepsilon}=\{x$ $\left.\in \Omega:\left|x_{3}\right| \leq \varepsilon \varphi\left(x^{\prime} / \varepsilon\right)\right\}$ and $x=\left(x^{\prime}, x_{3}\right), \Omega_{\varepsilon}=\Omega \backslash B_{\varepsilon}, \varepsilon$ be a positive small enough parameter and $\varphi$ be $a] 0,1[\times] 0,1[-$ periodic function. Let us consider a thermal problem on the body occupying the domain $\Omega$, where a very high heat conductivity on the layer $B_{\varepsilon}$ is considered. The last body mentioned is subject to an outside temperature $f, f: \Omega \longrightarrow \mathbb{R}$, and cooled at the boundary $\partial \Omega$. The problem is modeled with the following equations

$$
\left\{\begin{array}{l}
\Delta_{p(\cdot)} u^{\varepsilon}+f=0 \text { in } \Omega_{\mathcal{\varepsilon}} \\
\frac{1}{\mathcal{E}^{\alpha}} \Delta_{p(\cdot)} u^{\varepsilon}+f=0 \text { in } B_{\varepsilon}, \\
{\left[u^{\varepsilon}\right]=0 \text { on } \sum_{\varepsilon}^{ \pm},} \\
\left.\left|\nabla u^{\varepsilon}\right|^{p(\cdot)-2} \frac{\partial u^{\varepsilon}}{\partial n}\right|_{\Omega_{\varepsilon}}=\left.\frac{1}{\mathcal{E}^{\alpha}}\left|\nabla u^{\varepsilon}\right|^{p(\cdot)-2} \frac{\partial u^{\varepsilon}}{\partial n}\right|_{B_{\varepsilon}} \text { on } \sum_{\varepsilon}^{ \pm} \\
u^{\varepsilon}=0 \text { on } \partial \Omega,
\end{array}\right.
$$

the conductivity is expressed by $1 / \varepsilon^{\alpha}$ and the unknown $u^{\varepsilon}$ be the temperature, $n$ be the outward normal to $\sum_{\varepsilon}^{ \pm}, p()>1,. \alpha$ $>0, f \in L^{\infty}(\Omega)$, we denote by $[v]$ the jump of the function $v, \Delta_{p(\text {.) }}$ is $p($.$) -Laplace operator defined on sobolev space$ $W_{0}^{1, p(\cdot)}(\Omega)$ to its dual space $W_{0}^{-1, p(\cdot)}(\Omega)$. In setting of the constant exponent case, the asymptotic analysis of linear and nonlinear either in thermal and thermoelasticity problems with heat conductivity is widely treated by many authors, for example, in a structure with plate case, we can refer the reader to Ait Moussa and Licht in [3], Bourgeat and Tapiero in [4], Suquet and Sawczuck in [5], Brillard et al. in [6, 7], Pesqueira et al. in $[8,9]$, Silva et al. in $[10,11]$, Arrieta and Pereira in [12], and Sanchez-Palencia et al. in [13, 14], in other hand for a structure with an oscillating layer, Messaho et al. have studied the problem with very high conductivity in the layer in $[15,16]$ and Ameziane et al. have treated the case of a porous media behavior in [17]. Now, the aim of the present work is to study the problem of existence and limit behavior of a weak solutions for elliptic problem with variable exponent expressed by (1), which presents several mathematical difficulties either in terms of choosing a suitable Luxemburg norm for the considered Sobolev space or using the epiconvergence method to find the limit problem of our model. Accordingly, the limit behavior consist, via the epiconvergence method (see for instance $[18,19]$ ), to find a minimization limit problem linked to the minimization problem related to (1). Where $\varepsilon$ being a positive parameter intended to tend toward zero and $\varphi_{\varepsilon}$ is a bounded real function, $] 0, \varepsilon\left[^{2}\right.$-periodic.

This paper is organized in the following way. In Section 2, we give some notations and assumptions on the function $p($. , functional spaces for our study, and results for the epiconvergence notion (in appendix) that will be used throughout this paper. We study problem (5) and its limit asymptotic in Section 5.

\section{Notations and Assumptions}

In this section, we will give the notations that will be used throughout this paper:

(i) $\Omega$ be a bounded domain in $\mathbb{R}^{3}$ with meas $(\Omega)>0, x$ $=\left(x^{\prime}, x_{3}\right)$ where $x^{\prime}=\left(x_{1}, x_{2}\right)$

(ii) $Y=] 0,1[\times] 0,1\left[, \varphi: \mathbb{R}^{2} \longrightarrow\left[a_{1}, a_{2}\right]\right.$, where $\varphi$ is $Y$ -periodic and $a_{2} \geq a_{1}>0$

(iii) $\varphi_{\varepsilon}\left(x^{\prime}\right)=\varphi\left(x^{\prime} / \varepsilon\right), \quad m(\varphi)=1 / \operatorname{meas}(Y) \int_{Y} \varphi\left(x^{\prime}\right) d x^{\prime}$, $\eta(\alpha)=\lim _{\varepsilon \longrightarrow 0} \varepsilon^{1-\alpha}$ with $\alpha \geq 0$

(iv) $C$ will denote any constant with respect to $\varepsilon$ and $0 .+$ $\infty=0$

Now, we assume that the function $p($.$) and r$ satisfying the following conditions

$$
p(.) \in \mathscr{C}^{0}(\bar{\Omega}) \quad \text { with } \quad p(.)>1 \quad \text { in } \quad \bar{\Omega}
$$

there exists $\left.p^{-}, p^{+} \in\right] 1,3\left[\right.$ such that $p^{-}=\inf _{x \in \bar{\Omega}} p(x)$ and

$$
p^{+}=\sup _{x \in \bar{\Omega}} p(x)
$$

$\left\{\begin{array}{l}\text { there exists a continuous } \quad \omega: \mathbb{R}^{+} \longrightarrow \mathbb{R}^{+}, \\ \forall x, y \in \Omega,|p(x)-p(y)| \leq \omega(|x-y|) \quad \text { with } \quad \lim _{\tau 0} \omega(\tau) \log \left(\frac{1}{\tau}\right)=0 .\end{array}\right.$

\section{Main Results}

3.1. Study of the Problem (1). Note that problem (1) is equivalent to the minimization problem

$$
\begin{aligned}
& \inf _{v \in W_{0}^{1, p(\cdot)}(\Omega)}\left\{\Phi^{\varepsilon}(v)+\mathscr{F}(v)\right\}, \\
& \Phi^{\varepsilon}(v)=\int_{\Omega_{\varepsilon}} \frac{1}{p(x)}|\nabla v|^{p(x)} d x+\frac{1}{\varepsilon^{\alpha}} \int_{B_{\varepsilon}} \frac{1}{p(x)}|\nabla v|^{p(x)} d x, \\
& \mathscr{F}(v)=-\int_{\Omega} f . v d x .
\end{aligned}
$$

So, problems (1) and (5) have the same weak solutions in $W_{0}^{1, p(.)}(\Omega)$. Next, we will be interested to study the 
minimization problem (5), and the existence of its weak solutions is given in the following proposition.

Proposition 1. Assume that assumptions (2) and (3) hold. If $f \in L^{\infty}(\Omega)$, then, the problem (5) admits an unique solution $u^{\varepsilon}$ in $W_{0}^{1, p(.)}(\Omega)$.

The proof of this proposition is based on classical convexity arguments see for example [20,21].

In the sequel, we focus on the limit behavior of the solution $u^{\varepsilon}$ of problem (5) with respect to the values of $\alpha$. In order to establish this behavior, we use the epiconvergence method (see Definition B.1), to starting this, in one hand, we need to determine the space and its topology in applying the epiconvergence method. Now, we give in the following lemma, the estimations on $\nabla u^{\varepsilon}$.

Lemma 2. Assuming that there exists constants $C>0$ and $a$ sequence $\left(u^{\varepsilon}\right)_{\varepsilon>0} \subset W_{0}^{1, p(.)}(\Omega)$ such that $\Phi^{\varepsilon}\left(u^{\varepsilon}\right)+\mathscr{F}\left(u^{\varepsilon}\right) \leq C$, under (2), (3), and for $f \in L^{\infty}(\Omega)$. Then, $\left(u^{\varepsilon}\right)_{\varepsilon>0}$ satisfies to

$$
\begin{gathered}
\int_{\Omega_{\varepsilon}}\left|\nabla u^{\varepsilon}\right|^{p(x)} d x \leq C, \\
\int_{B_{\varepsilon}}\left|\nabla u^{\varepsilon}\right|^{p(x)} d x \leq C \varepsilon^{\alpha},
\end{gathered}
$$

Moreover, $u^{\varepsilon}$ is bounded in $W_{0}^{1, p(.)}(\Omega)$.

Proof. Since $u^{\varepsilon}$ satisfies to

$\int_{\Omega} \frac{1}{p(x)}\left|\nabla u^{\varepsilon}\right|^{p(x)} d x+\frac{1}{\varepsilon^{\alpha}} \int_{B_{\varepsilon}} \frac{1}{p(x)}\left|\nabla u^{\varepsilon}\right|^{p(x)} d x-\int_{\Omega} f u^{\varepsilon} d x \leq C$.

So, we have

$$
\int_{\Omega} \frac{1}{p(x)}\left|\nabla u^{\varepsilon}\right|^{p(x)} d x+\frac{1}{\varepsilon^{\alpha}} \int_{B_{\varepsilon}} \frac{1}{p(x)}\left|\nabla u^{\varepsilon}\right|^{p(x)} d x \leq C+\int_{\Omega} f u^{\varepsilon} d x .
$$
obtain

According to Hölder and Friedrich's inequalities, we

$$
\int_{\Omega} f u^{\varepsilon} d x \leq 2\|f\|_{L^{p(\cdot)}(\Omega)}\left\|u^{\varepsilon}\right\|_{L^{p(\cdot)}(\Omega)} \leq C\left\|\nabla u^{\varepsilon}\right\|_{L^{p(\cdot)}(\Omega)} .
$$

From (A.3) and Young inequality, we have

$$
\begin{aligned}
\int_{\Omega} f u^{\varepsilon} & \leq C \max \left(\left(A_{\Omega, p(.)}\left(\nabla u^{\varepsilon}\right)\right)^{1 / p^{-}},\left(A_{\Omega, p(.)}\left(\nabla u^{\varepsilon}\right)\right)^{1 / p^{+}}\right) \\
& \leq C+\max \left(\left(\frac{1}{p^{-}} A_{\Omega, p(.)}\left(\nabla u^{\varepsilon}\right)\right),\left(\frac{1}{p^{+}} A_{\Omega, p(.)}\left(\nabla u^{\varepsilon}\right)\right)\right) \\
& \leq C+\frac{1}{p^{-}} A_{\Omega, p(.)}\left(\nabla u^{\varepsilon}\right) \\
& \leq C+\frac{1}{p^{-}} \int_{\Omega_{\varepsilon}}\left|\nabla u^{\varepsilon}\right|^{p(x)} d x+\frac{1}{p^{-}} \int_{B_{\varepsilon}}\left|\nabla u^{\varepsilon}\right|^{p(x)} d x \\
& \leq C+\frac{1}{p^{-}} \int_{\Omega_{\varepsilon}}\left|\nabla u^{\varepsilon}\right|^{p(x)} d x+\frac{1}{\varepsilon^{\alpha} p^{-}} \int_{B_{\varepsilon}}\left|\nabla u^{\varepsilon}\right|^{p(x)} d x .
\end{aligned}
$$

From (11), we have

$$
\begin{aligned}
& \int_{\Omega_{\varepsilon}} \frac{1}{p(x)}\left|\nabla u^{\varepsilon}\right|^{p(x)} d x+\frac{1}{\varepsilon^{\alpha}} \int_{B_{\varepsilon}} \frac{1}{p(x)}\left|\nabla u^{\varepsilon}\right|^{p(x)} d x \\
& \leq C+\frac{1}{p^{-}} \int_{\Omega_{\varepsilon}}\left|\nabla u^{\varepsilon}\right|^{p(x)} d x+\frac{1}{\varepsilon^{\alpha} p^{-}} \int_{B_{\varepsilon}}\left|\nabla u^{\varepsilon}\right|^{p(x)} d x .
\end{aligned}
$$

So

$$
\frac{1}{\left(p^{-}\right)^{\prime}} \int_{\Omega_{\varepsilon}} \frac{1}{p(x)}\left|\nabla u^{\varepsilon}\right|^{p(x)} d x+\frac{1}{\left(p^{-}\right)^{\prime}} \frac{1}{\varepsilon^{\alpha}} \int_{B_{\varepsilon}} \frac{1}{p(x)}\left|\nabla u^{\varepsilon}\right|^{p(x)} d x \leq C,
$$

from (3), we have $p(x) \leq p^{+}$in $\Omega$, so that

$$
\frac{1}{p^{+}\left(p^{-}\right)^{\prime}} \int_{\Omega_{\varepsilon}}\left|\nabla u^{\varepsilon}\right|^{p(x)} d x+\frac{1}{p^{+}\left(p^{-}\right)^{\prime}} \frac{1}{\varepsilon^{\alpha}} \int_{B_{\varepsilon}}\left|\nabla u^{\varepsilon}\right|^{p(x)} d x \leq C .
$$

Therefore, we will have the assertions (8), (9), and from (A.3), we deduce that $\left(u^{\varepsilon}\right)$ is bounded in $W_{0}^{1, p(.)}(\Omega)$.

As a consequence, we can confirm that the solution of problem (5) satisfies Lemma 2.

Now, in the following proposition, we will discuss, according to the real values of $\alpha$, to obtain some information about the behavior of solution $\left(u^{\varepsilon}\right)_{\varepsilon}$ of problem (5) when $\varepsilon$ close to zero, for this, we need to define an operator which transforms the functions defined on $B_{\varepsilon}$ to the functions defined on $\Sigma$, like in [15], let us define the following operator $m^{\varepsilon}: W^{1, p(.)}\left(B_{\varepsilon}\right) \longrightarrow W^{1, p(.)}(\Sigma)$, by

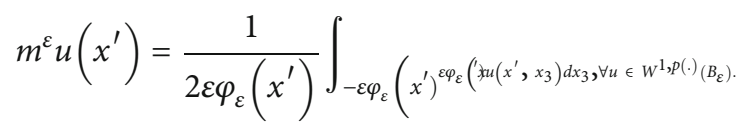

This mean operator satisfies the following results:

Lemma 3. The operator $m^{\varepsilon}$ definite by (17) is linear and bounded of $L^{p(.)}\left(B_{\varepsilon}\right)$ (respectively, $W^{1, p(.)}\left(B_{\varepsilon}\right)$ ) in $L^{p(.)}(\Sigma)$ 
(respectively, $W^{1, p(.)}(\Sigma)$ ), with norm $\leq C \varepsilon^{-1 / p^{-}}$, moreover, for all $u \in W^{1, p(.)}\left(B_{\varepsilon}\right)$, we have

$$
\int_{\Sigma}\left|m^{\varepsilon} u-u_{\left.\right|_{\Sigma}}\right|^{p(x)} \leq C \varepsilon^{p^{-}-1} \int_{B_{\varepsilon}}|\nabla u|^{p(x)} .
$$

Lemma 4. Let $\left(u^{\varepsilon}\right)_{\varepsilon>0} \subset W_{0}^{1, p(.)}(\Omega)$ which satisfies (8) and (9), then

$$
\int_{\Sigma}\left|\nabla^{\prime}\left(m^{\varepsilon} u^{\varepsilon}\right)\right|^{p(x)} \leq C \varepsilon^{\alpha-1}
$$
$(\Sigma)$.

Moreover, $m^{\varepsilon} u^{\varepsilon}$ possesses a bounded subsequence in $L^{p(.)}$

The proof of these two previous lemmas is similar to the lemmas 4.2 and 4.3 in the reference [15].

Proposition 5. The solution of the problem (5), $\left(u^{\varepsilon}\right)_{\mathcal{\varepsilon}}$, possesses a subsequence denoted also $\left(u^{\varepsilon}\right)_{\varepsilon}$ weakly convergent toward an element $u^{*}$ in $W_{0}^{1, p(.)}(\Omega)$ satisfying

$$
\begin{gathered}
u_{\left.\right|_{\Sigma}} \in L^{p(\cdot)}(\Sigma), \\
\text { if } \alpha=1,\left.u^{*}\right|_{\Sigma} \in W^{1, p(\cdot)}(\Sigma), \\
\forall \alpha>1,\left.u^{*}\right|_{\left.\right|_{\Sigma}}=C .
\end{gathered}
$$

Proof. Given that the solution of problem (5) satisfies Lemma 2 and according to the same lemma, then, the sequence $u^{\varepsilon}$ is bounded in $W_{0}^{1, p(.)}(\Omega)$, it follows that there exists an element $u^{*} \in W_{0}^{1, p(.)}(\Omega)$ and a subsequence of $u^{\varepsilon}$, still denoted by $u^{\varepsilon}$ such that $u^{\varepsilon} \rightarrow u^{*}$ in $W_{0}^{1, p(.)}(\Omega)$. Under (2), (3), and (4), the trace operator proprieties holds in the Sobolev space with variable exponent, for more details, see for example [22], so

$$
u_{\left.\right|_{\Sigma}}^{\varepsilon} \rightarrow u^{*}{ }_{\left.\right|_{\Sigma}} \text { in } L^{p(\cdot)}(\Sigma)
$$

thanks to Lemma 3, we have

$$
\int_{\Sigma}\left|m^{\varepsilon} u^{\varepsilon}-u_{\left.\right|_{\Sigma}}^{\varepsilon}\right|^{p(x)} \leq C \varepsilon^{p^{-}-1} \int_{B_{\varepsilon}}\left|\nabla u^{\varepsilon}\right|^{p(x)},
$$

and according to (9), we have

$$
\int_{\Sigma}\left|m^{\varepsilon} u^{\varepsilon}-u_{\left.\right|_{\Sigma}}^{\varepsilon}\right|^{p(x)} \leq C \varepsilon^{\alpha+p^{-}-1} .
$$

For $\alpha=1$, according to the evaluation (19), the sequence $\nabla^{\prime} m^{\varepsilon} u^{\varepsilon}$ possesses a subsequence, still denoted by $\nabla^{\prime} m^{\varepsilon} u^{\varepsilon}$ weakly convergent to an element $u^{2}$ in $L^{p(.)}(\Sigma)^{2}$, as $m^{\varepsilon} u^{\varepsilon}-$ $u^{*}{ }_{\left.\right|_{\Sigma}}$ in $L^{p(.)}(\Sigma)$, so one concludes that $m^{\varepsilon} u^{\varepsilon} \rightarrow u^{*}{ }_{\left.\right|_{\Sigma}}$ in $W^{1, p(.)}$ $(\Sigma)$ and $\nabla^{\prime} u_{\left.\right|_{\Sigma}}^{*}=u^{2}$. Hence, $u_{\left.\right|_{\Sigma}}^{*} \in W^{1, p(.)}(\Sigma)$.

For $\alpha>1$, one shows, as in the case $\alpha=1$ and taking $u^{2}$ $=0$, that $\left.u^{*}\right|_{\Sigma}=C$.
The limit behavior of the problem (5) will be derived with the epiconvergence method (see Definition B.1).

3.2. Limit Behavior of the Problem (5). In this subsection, we will interest, according to the values of $\alpha$, to find the limit problem of the problem (5). Consider the following energy functional given by

$$
\mathscr{F}^{\varepsilon}(u)=\int_{\Omega_{\varepsilon}} \frac{1}{p(x)}|\nabla u|^{p(x)}+\frac{1}{\varepsilon^{\alpha}} \int_{B_{\varepsilon}} \frac{1}{p(x)}|\nabla u|^{p(x)}, \forall u \in W^{1, p(.)}(\Omega),
$$

$$
\Phi(u)=-\int_{\Omega} f u, \forall u \in W^{1, p(\cdot)}(\Omega) .
$$

We denote by $\tau_{f}$ the weak topology on $W^{1, p(.)}(\Omega)$ and let

$$
\begin{gathered}
V(\Sigma)=\left\{u \in W_{0}^{1, p(\cdot)}(\Omega):\left.u\right|_{\Sigma} \in W^{1, p(\cdot)}(\Sigma)\right\}, \\
V^{C}(\Sigma)=\left\{u \in W_{0}^{1, p(\cdot)}(\Omega):\left.u\right|_{\Sigma}=C\right\} .
\end{gathered}
$$

The set $V^{C}(\Sigma)$ is a Banach space with the norm of $W_{0}^{1, p(.)}(\Omega)$. We show easily that $V(\Sigma)$ is a Banach space with the norm

$$
u \mapsto\|\nabla u\|_{L^{p(\cdot)}(\Omega)^{3}}+\left\|\nabla^{\prime} u_{\left.\right|_{\Sigma}}\right\|_{L^{p(x)}(\Sigma)^{2}}
$$

Let

$\mathbb{G}^{\alpha}=\left(\begin{array}{lll}\left\{u \in W_{0}^{1, p(\cdot)}(\Omega):\left.\eta(\alpha) u\right|_{\Sigma} \in W^{1, p(.)}(\Sigma)\right\} & \text { if } & \alpha \leq 1, \\ V^{C} & \text { if } & \alpha>1 .\end{array}\right.$

$$
\mathbb{D}^{\alpha}=\left(\begin{array}{lll}
\mathscr{D}(\Omega) & \text { if } & \alpha \leq 1, \\
\left\{u \in \mathscr{D}(\Omega):\left.u\right|_{\Sigma}=C\right\} & \text { if } & \alpha>1 .
\end{array}\right.
$$

It is known that $\overline{\mathbb{D}}^{\alpha}=\mathbb{G}^{\alpha}$.

Theorem 6. According to the values of $\alpha$, there exists a functional $F^{\alpha}$ defined on $W_{0}^{1, p(.)}(\Omega)$ with value in $\mathbb{R} \cup\{+\infty\}$ such that $\tau_{f}-\lim _{e} \mathscr{F}^{\varepsilon}=F^{\alpha}$ in $W_{0}^{1, p}(\Omega)$, where the functional $F^{\alpha}$ is given as follows

If $0 \leq \alpha<1$ :

$$
F^{\alpha}(u)=\int_{\Omega} \frac{1}{p(x)}|\nabla u|^{p(x)}, \forall u \in W_{0}^{1, p(.)}(\Omega) .
$$




$$
\text { If } \alpha \geq 1 \text { : }
$$

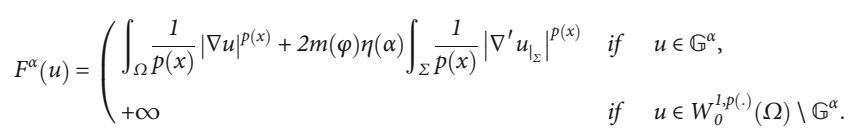

Proof.

(a) One Is Going to Determine the Upper Epilimit. Let $u$ $\in \mathbb{G}^{\alpha} \subset W_{0}^{1, p(.)}(\Omega)$, there exists a sequence $\left(u^{n}\right)$ in $\mathbb{D}^{\alpha}$ such that

$$
u^{n} \longrightarrow u \quad \text { in } \quad \mathbb{G}^{\alpha} \text {, when } n \longrightarrow+\infty
$$

So that $u^{n} \longrightarrow u$ in $W_{0}^{1, p(\cdot)}(\Omega)$. Let $\theta$ be a smooth function satisfying

$\theta(t)=1 \quad$ if $\quad|t| \leq 1, \theta(t)=0 \quad$ if $\quad|t| \geq 2 \quad$ and $\quad\left|\theta^{\prime}(t)\right| \leq 2 \forall t \in \mathbb{R}$,

and set

$$
\theta_{\varepsilon}(x)=\theta\left(\frac{x_{3}}{\varepsilon \varphi_{\varepsilon}\left(x^{\prime}\right)}\right)
$$

we define

$$
u^{\varepsilon, n}=\theta_{\varepsilon}(x) u_{{ }_{\Sigma}}^{n}+\left(1-\theta_{\varepsilon}(x)\right) u^{n},
$$

One shows easily that $u^{\varepsilon, n} \in W_{0}^{1, p(.)}(\Omega)$ and $u^{\varepsilon, n} \longrightarrow u^{n}$ in $\mathbb{G}^{\alpha}$, when $\varepsilon \longrightarrow 0$. Since

$$
\mathscr{F}^{\varepsilon}\left(u^{\varepsilon, n}\right)=\int_{\Omega_{\varepsilon}} \frac{1}{p(x)}\left|\nabla u^{\varepsilon, n}\right|^{p(x)}+\frac{1}{\varepsilon^{\alpha}} \int_{B_{\varepsilon}} \frac{1}{p(x)}\left|\nabla u^{\varepsilon, n}\right|^{p(x)},
$$

so that

$$
\begin{aligned}
\mathscr{F}^{\varepsilon}\left(u^{\varepsilon, n}\right)= & \int_{\left|x_{3}\right|>2 \varepsilon \varphi_{\varepsilon}} \frac{1}{p(x)}\left|\nabla u^{\varepsilon, n}\right|^{p(x)}+\int_{\varepsilon \varphi_{\varepsilon}<\left|x_{3}\right|<2 \varepsilon \varphi_{\varepsilon}} \frac{1}{p(x)}\left|\nabla u^{\varepsilon, n}\right|^{p(x)} \\
& +\frac{1}{\varepsilon^{\alpha}} \int_{B_{\varepsilon}} \frac{1}{p(x)}\left|\nabla u^{\varepsilon, n}\right|^{p(x)} \\
= & \int_{\left|x_{3}\right|>2 \varepsilon \varphi_{\varepsilon}} \frac{1}{p(x)}\left|\nabla u^{n}\right|^{p(x)}+\int_{\varepsilon \varphi_{\varepsilon}<\left|x_{3}\right|<2 \varepsilon \varphi_{\varepsilon}} \frac{1}{p(x)}\left|\nabla u^{\varepsilon, n}\right|^{p(x)} \\
& +\left.2 \varepsilon^{1-\alpha} \int_{\Sigma} \varphi_{\varepsilon} \frac{1}{p(x)}\left|\nabla^{\prime} u^{n}\right|_{\Sigma}\right|^{p(x)} .
\end{aligned}
$$

Since $\varphi_{\varepsilon}$ is bounded, one verifies easily that

$$
\lim _{\varepsilon \longrightarrow 0}\left\{\int_{\varepsilon \varphi_{\varepsilon}<\left|x_{3}\right|<2 \varepsilon \varphi_{\varepsilon}} \frac{1}{p(x)}\left|\nabla u^{\varepsilon, n}\right|^{p(x)}\right\}=0 .
$$

(1) If $\alpha \leq 1$. Since $\varphi_{\varepsilon}-{ }^{*} m(\varphi)$ in $L^{\infty}(\Sigma)$ and $\varepsilon^{1-\alpha} \longrightarrow \eta$ $(\alpha)$, it follows that

$$
\left.\lim _{\varepsilon \rightarrow 0} 2 \varepsilon^{1-\alpha} \int_{\Sigma} \varphi_{\varepsilon} \frac{1}{p(x)}\left|\nabla^{\prime} u^{n}\right|_{\Sigma}\right|^{p(x)}=\left.2 m(\varphi) \eta(\alpha) \int_{\Sigma} \frac{1}{p(x)}\left|\nabla^{\prime} u^{n}\right|_{\Sigma}\right|^{p(x)} .
$$

By passing to the upper limit, one has

$$
\begin{aligned}
& \limsup _{\varepsilon \rightarrow 0} \mathscr{F}^{\varepsilon}\left(u^{\varepsilon, n}\right) \\
& \quad=\limsup _{\varepsilon \longrightarrow 0}\left(\int_{\left|x_{3}\right|>2 \varepsilon \varphi_{\varepsilon}} \frac{1}{p(x)}\left|\nabla u^{n}\right|^{p(x)}+\left.2 \varepsilon^{1-\alpha} \int_{\Sigma} \varphi_{\varepsilon} \frac{1}{p(x)}\left|\nabla^{\prime} u^{n}\right|_{\Sigma}\right|^{p(x)}\right) \\
& \quad=\int_{\Omega} \frac{1}{p(x)}\left|\nabla u^{n}\right|^{p(x)}+2 m(\varphi) \eta(\alpha) \int_{\Sigma} \frac{1}{p(x)}\left|\nabla^{\prime} u^{n}\right|_{\Sigma}^{p(x)}
\end{aligned}
$$

(2) If $\alpha>1$. By passing to the upper limit, one has

$$
\begin{aligned}
\limsup _{\varepsilon \longrightarrow 0} \mathscr{F}^{\varepsilon}\left(\mathcal{u}^{\varepsilon, n}\right) & =\limsup _{\varepsilon \longrightarrow 0}\left(\int_{\left|x_{3}\right|>2 \varepsilon \varphi_{\varepsilon}} \frac{1}{p(x)}\left|\nabla u^{n}\right|^{p(x)}\right) \\
& =\int_{\Omega} \frac{1}{p(x)}\left|\nabla u^{n}\right|^{p(x)} .
\end{aligned}
$$

Since $u^{n} \longrightarrow u$ in $\mathbb{G}^{\alpha}$, when $n \longrightarrow+\infty$. According to the classical result, diagonalization's lemma ([18], Lemma $1.15)$, there exists a function $n(\varepsilon): \mathbb{R}^{+} \longrightarrow \mathbb{N}$ increasing to $+\infty$ when $\varepsilon \longrightarrow 0$, such that $u^{\varepsilon, n(\varepsilon)} \longrightarrow u$ in $\mathbb{G}^{\alpha}$ when $\varepsilon \longrightarrow$ 0 . While $n$ approaches $+\infty$, one will have

(1) If $\alpha \neq 1$

$$
\limsup _{\varepsilon \longrightarrow 0} \mathscr{F}^{\varepsilon}\left(u^{\varepsilon, n(\varepsilon)}\right) \leq \operatorname{limsuplimsup}_{n \longrightarrow+\infty} \mathscr{F}_{\varepsilon \longrightarrow 0}\left(u^{\varepsilon, n}\right) \leq \int_{\Omega} \frac{1}{p(x)}|\nabla u|^{p(x)} .
$$

(2) If $\alpha=1$ 


$$
\begin{aligned}
\limsup _{\varepsilon \longrightarrow 0} \mathscr{F}^{\varepsilon}\left(u^{\varepsilon, n(\varepsilon)}\right) & \leq \operatorname{limsuplimsup}_{n \longrightarrow+\infty} \mathscr{F}^{\varepsilon}\left(u^{\varepsilon, n}\right) \\
& \leq \int_{\Omega} \frac{1}{p(x)}|\nabla u|^{p(x)}+2 m(\varphi) \eta(\alpha) \int_{\Sigma} \frac{1}{p(x)}\left|\nabla^{\prime} u_{\left.\right|_{\Sigma}}\right|^{p(x)} .
\end{aligned}
$$

If $u \in W_{0}^{1, p(.)}(\Omega) \backslash \mathbb{G}^{\alpha}$, it is clear that, for every $u^{\varepsilon} \in$ $W_{0}^{1, p(.)}(\Omega), u^{\varepsilon} \rightarrow u$ in $W_{0}^{1, p(.)}(\Omega)$, one has

$$
\limsup _{\varepsilon \longrightarrow 0} \mathscr{F}^{\varepsilon}\left(u^{\varepsilon}\right) \leq+\infty \text {. }
$$

(b) One is going to determine the Lower Epilimit. Let $u$ $\in \mathbb{G}^{\alpha}$ and $\left(u^{\varepsilon}\right)$ be a sequence in $W_{0}^{1, p(.)}(\Omega)$ such that $u^{\varepsilon} \rightarrow u$ in $W_{0}^{1, p(.)}(\Omega)$, so that

$$
\chi_{\Omega_{\varepsilon}} \nabla u^{\varepsilon} \rightarrow \nabla u \quad \text { in } \quad L^{p(\cdot)}(\Omega)^{3} .
$$

(1) If $\alpha \neq 1$. Since

$$
\mathscr{F}^{\varepsilon}\left(u^{\varepsilon}\right) \geq \int_{\Omega_{\varepsilon}} \frac{1}{p(x)}\left|\nabla u^{\varepsilon}\right|^{p(x)}
$$
obtains

According to (5) and by passing to the lower limit, one

$$
\liminf _{\varepsilon \rightarrow 0} \mathscr{F}^{\varepsilon}\left(u^{\varepsilon}\right) \geq \int_{\Omega} \frac{1}{p(x)}|\nabla u|^{p(x)}
$$

(2) If $\alpha=1$. If $\lim \inf _{\varepsilon \longrightarrow 0} \mathscr{F}^{\varepsilon}\left(u^{\varepsilon}\right)=+\infty$, there is nothing to prove, because

$$
\int_{\Omega} \frac{1}{p(x)}|\nabla u|^{p(x)}+2 m(\varphi) \eta(\alpha) \int_{\Sigma} \frac{1}{p(x)}\left|\nabla^{\prime} u_{\left.\right|_{\Sigma}}\right|^{p(x)} \leq+\infty
$$

Otherwise, $\lim \inf _{\varepsilon \rightarrow 0} \mathscr{F}^{\varepsilon}\left(u^{\varepsilon}\right)<+\infty$, there exists a subsequence of $\mathscr{F}^{\varepsilon}\left(u^{\varepsilon}\right)$ still denoted by $\mathscr{F}^{\varepsilon}\left(u^{\varepsilon}\right)$ and a constant $C$ $>0$, such that $\mathscr{F}^{\varepsilon}\left(u^{\varepsilon}\right) \leq C$, which implies that

$$
\frac{1}{\mathcal{E}^{\alpha}} \int_{B_{\varepsilon}} \frac{1}{p(x)}\left|\nabla u^{\varepsilon}\right|^{p(x)} \leq C .
$$

So $u^{\varepsilon}$ satisfies the hypothesis of the Lemma 4 , and according to this last, $\nabla^{\prime} m^{\varepsilon} u^{\varepsilon}$ is bounded in $L^{p(.)}(\Sigma)^{2}$, so there exists an element $u_{1} \in L^{p(.)}(\Sigma)^{2}$ and a subsequence of $\nabla^{\prime} m^{\varepsilon} u^{\varepsilon}$, still denoted by $\nabla^{\prime} m^{\varepsilon} u^{\varepsilon}$, such that $\nabla^{\prime} m^{\varepsilon} u^{\varepsilon} \longrightarrow u_{1}$ in $L^{p(.)}(\Sigma)^{2}$, since $u_{\left.\right|_{\Sigma}}^{\varepsilon} \rightarrow u_{\left.\right|_{\Sigma}}$ in $L^{p(.)}(\Sigma)$, and thanks to (18) and (50), one has $m^{\varepsilon} u^{\varepsilon} \rightarrow u_{\left.\right|_{\Sigma}}$ in $L^{p(.)}(\Sigma)$, then $m^{\varepsilon} u^{\varepsilon} \rightarrow u_{\left.\right|_{\Sigma}}$ in $W^{1, p(.)}(\Sigma)$, therefore, $u_{1}=\nabla^{\prime} u_{\left.\right|_{\Sigma}}$, so that $\nabla^{\prime} m^{\varepsilon} u^{\varepsilon} \rightarrow \nabla^{\prime} u_{\left.\right|_{\Sigma}}$ in $L^{p(.)}(\Sigma)^{2}$. One has

$$
\begin{aligned}
\mathscr{F}^{\varepsilon}\left(u^{\varepsilon}\right) & \geq \int_{\Omega_{\varepsilon}} \frac{1}{p(x)}\left|\nabla u^{\varepsilon}\right|^{p(x)}+\frac{1}{\varepsilon^{\alpha}} \int_{B_{\varepsilon}} \frac{1}{p(x)}\left|\nabla u^{\varepsilon}\right|^{p(x)} \\
& \geq \int_{\Omega_{\varepsilon}} \frac{1}{p(x)}\left|\nabla u^{\varepsilon}\right|^{p(x)}+2 \varepsilon^{1-\alpha} \int_{\Sigma} \varphi_{\varepsilon} \frac{1}{p(x)}\left|\nabla^{\prime} m^{\varepsilon} u^{\varepsilon}\right|^{p(x)} .
\end{aligned}
$$

Using the subdifferential inequality of

$$
v \longrightarrow 2 \varepsilon^{1-\alpha} \int_{\Sigma} \frac{\varphi_{\varepsilon}}{p(x)}|v|^{p(x)}, \forall v \in L^{p(.)}(\Sigma)^{2},
$$

one has

$$
\begin{aligned}
\mathscr{F}^{\varepsilon}\left(u^{\varepsilon}\right) \geq & \int_{\Omega_{\varepsilon}} \frac{1}{p(x)}\left|\nabla u^{\varepsilon}\right|^{p(x)}+2 \varepsilon^{1-\alpha} \int_{\Sigma} \varphi_{\varepsilon} \frac{1}{p(x)}\left|\nabla^{\prime} u_{\left.\right|_{\Sigma}}\right|^{p(x)} \\
& +2 \varepsilon^{1-\alpha} \int_{\Sigma} \varphi_{\varepsilon}\left|\nabla^{\prime} u_{\left.\right|_{\Sigma}}\right|^{p(x)-2} \nabla^{\prime} u_{\left.\right|_{\Sigma}}\left(\nabla^{\prime} m^{\varepsilon} u^{\varepsilon}-\nabla^{\prime} u_{\left.\right|_{\Sigma}}\right) .
\end{aligned}
$$

Thanks to Lemma B.4, one has $\varphi_{\varepsilon} \longrightarrow m(\varphi)$ in $L^{p^{\prime}(.)}(\Sigma)$, so according to (5) and by passing to the lower limit, one obtains

$$
\liminf _{\varepsilon \longrightarrow 0} \mathscr{F}^{\varepsilon}\left(u^{\varepsilon}\right) \geq \int_{\Omega} \frac{1}{p(x)}|\nabla u|^{p(x)}+2 m(\varphi) \eta(\alpha) \int_{\Sigma} \frac{1}{p(x)}\left|\nabla^{\prime} u_{\left.\right|_{\Sigma}}\right|^{p(x)}
$$

If $u \in W_{0}^{1, p(.)}(\Omega) \backslash \mathbb{G}^{\alpha}$ and $u^{\varepsilon} \in W_{0}^{1, p(.)}(\Omega)$, such that $u^{\varepsilon}$ $\rightarrow u$ in $W_{0}^{1, p(.)}(\Omega)$.

Assume that

$$
\liminf _{\varepsilon \longrightarrow 0} \mathscr{F}^{\varepsilon}\left(u^{\varepsilon}\right)<+\infty
$$

So there exists a constant $C>0$ and a subsequence of $\mathscr{F}^{\varepsilon}\left(u^{\varepsilon}\right)$, still denoted by $\mathscr{F}^{\varepsilon}\left(\mathcal{u}^{\varepsilon}\right)$, such that

$$
\mathscr{F}^{\varepsilon}\left(u^{\varepsilon}\right)<C .
$$

For $0 \leq \alpha<1$, there is nothing to prove.

Otherwise, one takes the same way as in the case $u \in \mathbb{G}^{\alpha=1}$, one has $\nabla^{\prime} m^{\varepsilon} u^{\varepsilon}$ is bounded in $L^{p(.)}(\Sigma)^{2}$, so there exists an element $u_{1} \in L^{p(.)}(\Sigma)^{2}$ and a subsequence of $\nabla^{\prime} m^{\varepsilon} u^{\varepsilon}$, still denoted by $\nabla^{\prime} m^{\varepsilon} u^{\varepsilon}$, such that $\nabla^{\prime} m^{\varepsilon} u^{\varepsilon} \rightarrow u_{1}$ in $L^{p(.)}(\Sigma)^{2}$, since $u_{\left.\right|_{\Sigma}}^{\varepsilon} \rightarrow u_{\left.\right|_{\Sigma}}$ in $L^{p(.)}(\Sigma)$, and thanks to (18) and (56), one has $m^{\varepsilon} u^{\varepsilon} \rightarrow u_{\left.\right|_{\Sigma}}$ in $L^{p(.)}(\Sigma)$, then $m^{\varepsilon} u^{\varepsilon} \rightarrow u_{\left.\right|_{\Sigma}}$ in $W^{1, p(.)}(\Sigma)$, so that $u \in \mathbb{G}^{\alpha}$ what contradicts the fact that $u \in \mathbb{G}^{\alpha}$, so that

$$
\liminf _{\varepsilon \longrightarrow 0} \mathscr{F}^{\varepsilon}\left(u^{\varepsilon}\right)=+\infty
$$

Hence, the proof of Theorem 6 is complete. 
In the sequel, one is interested to limit problem determination partner to the problem (5), when $\varepsilon$ approaches zero. Thanks to the epiconvergence results, (see Theorem B.3, Proposition B.2), Theorem 6 , and according to $\tau_{f}$-continuity of $\Phi$ in $W_{0}^{1, p(.)}(\Omega)$, one has $\mathscr{F}^{\varepsilon}+\Phi \tau_{f}$ - epilimit toward $\mathscr{F}_{\alpha}+\Phi$ in $W_{0}^{1, p(.)}(\Omega)$.

Proposition 7. For every $f \in L^{\infty}(\Omega)$ and according to the parameter values of $\alpha$, there exists $u^{*} \in W_{0}^{1, p(.)}(\Omega)$ satisfying

$$
\begin{gathered}
u^{\varepsilon}-u^{*} \quad \text { in } W_{0}^{1, p(.)}(\Omega), \\
F^{\alpha}\left(u^{*}\right)+\Phi\left(u^{*}\right)=\inf _{v \in \mathbb{G}^{\alpha}}\left\{F^{\alpha}(v)+\Phi(v)\right\} .
\end{gathered}
$$

Proof. Thanks to Lemma 2, the family $\left(u^{\varepsilon}\right)$ is bounded in $W_{0}^{1, p(.)}(\Omega)$, therefore, it possess a $\tau_{f}-$ cluster point $u^{*}$ in $W_{0}^{1, p(.)}(\Omega)$. And thanks to a classical epiconvergence result (see Theorem B.3), one has $u^{*}$ is a solution of the problem find

$$
\inf _{v \in W_{0}^{1, p(.)}(\Omega)}\left\{\mathscr{F}_{\alpha}(v)+\Phi(v)\right\}
$$

Since for $\alpha>1, \mathscr{F}_{\alpha}$ equals $+\infty$ on $W_{0}^{1, p(.)}(\Omega) \backslash \mathbb{G}^{\alpha},(60)$ becomes

$$
\inf _{v \in \mathbb{G}^{\alpha}}\left\{\mathscr{F}_{\alpha}(v)+\Phi(v)\right\}
$$

According to the uniqueness of solutions of the problem (8), so that $u^{\varepsilon}$ admits an unique $\tau_{f}$-cluster point $u^{*}$, and therefore, $u^{\varepsilon} \rightarrow u^{*}$ in $W_{0}^{1, p(.)}(\Omega)$.

Remark 8. One shows that the limit behavior of a constituted structure of two mediums of constant conductivity united by an oscillating nonlinear nanolayer of thickness $\varepsilon$, which the conductivity depends on the negative powers of $\varepsilon$, is described by a problem with interface $\Sigma$ ( $\Sigma$ the middle interface of the nanolayer). Following the powers of $\varepsilon$, to the inter- face $\Sigma$, one has, on the interface $\Sigma$ (the heat continuity), a bidimensional problem or the constant heat.

\section{Conclusion}

In this study, we have found out the limit behavior of weak solutions of the problem with variable exponent, of a containing structure, an oscillating nanolayer of thickness, and periodicity parameter depending of $\varepsilon$. The epiconvergence method, which is extremely popular in the last years and which has been crucial for the developments of the calculus of variations and of the theory of partial differential equations, is considered to find the limit problems with interface conditions. The bounds and the behavior of the solution are discussed and the obtained results agree with the results obtained in some previous works where the exponent is constant in homogenization theory. In future works, we extend the proposed scheme elliptic problem with variable exponent with nonhomogenous interfaces conditions in a nanostructure.

\section{Appendix}

\section{A. Sobolev Spaces with Variable Exponents}

In this section, we introduce the function spaces used throughout the paper and describe their basic properties, see for instance [22-24].

(1) By $L^{p(.)}(\Omega)$, we denote the space of measurable functions $u$ in $\Omega$ such that

$$
A_{\Omega, p(.)}(u)=\int_{\Omega}|u|^{p(x)} d x<+\infty .
$$

The space $L^{p(.)}(\Omega)$ equipped with the norm

$$
\|u\|_{L^{p(.)}(\Omega)}=\inf \left\{\lambda>0: A_{\Omega, p(.)}\left(\frac{u}{\lambda}\right) \leq 1\right\},
$$

becomes a Banach space.

(2) $\forall u \in L^{p(.)}(\Omega)$, the following inequalities hold

$$
\left\{\begin{array}{l}
\min \left(\|u\|_{L^{p(.)}(\Omega)}^{p^{-}},\|u\|_{L^{p(.)}(\Omega)}^{p^{+}}\right) \leq A_{\Omega, p(.)}(u) \leq \max \left(\|u\|_{L^{p^{(.)}(\Omega)}}^{p^{-}},\|u\|_{L^{p(.)}(\Omega)}^{p^{+}}\right) \\
\min \left(\left(A_{\Omega, p(.)}(u)\right)^{1 / p^{-}},\left(A_{\Omega, p(.)}(u)\right)^{1 / p^{+}}\right) \leq\|u\|_{L^{p(.)}(\Omega)} \leq \max \left(\left(A_{\Omega, p(.)}(u)\right)^{1 / p^{-}},\left(A_{\Omega, p(.)}(u)\right)^{1 / p^{+}}\right) .
\end{array}\right.
$$

(3) Let $p(),. q(.) \in \mathscr{C}(\bar{\Omega})$ satisfying the assumption (3), for $u \in L^{p(.)}(\Omega)$ and $v \in L^{q(.)}(\Omega)$ with $1 / p(x)+1 / q(x$ )$=1$ in $\Omega$, then the Holder's inequality holds

$$
\int_{\Omega} u v d x \leq 2\|u\|_{L^{p(\cdot)}(\Omega)}\|v\|_{L^{q(\cdot)}(\Omega)} .
$$


(4) According to (A.4), for every $1 \leq q=$ const $<p^{-} \leq p$ ( $x)<+\infty$

$\|u\|_{L^{q}(\Omega)} \leq 2 C\|u\|_{L^{p^{(.)}(\Omega)}} \quad$ with the constant $\quad C=\|1\|_{L^{p(\cdot) /(\cdot)-q}(\Omega)}$.

It is straightforward to check that for domain $\Omega$ such that $|\Omega|=\operatorname{meas}(\Omega)<+\infty$

$$
\|1\|_{L^{p(\cdot)}(\Omega)} \leq \max \left(|\Omega|^{1 / p^{-}},|\Omega|^{1 / p^{+}}\right)
$$

(5) The space $W^{1, p(.)}(\Omega)$, with $p($.$) satisfies to (2) and (3),$ is defined by

$$
W^{1, p(\cdot)}(\Omega)=\left\{u \in L^{p(\cdot)}(\Omega):|\nabla u| \in L^{p(\cdot)}(\Omega)\right\} .
$$

The space $W^{1, p(.)}(\Omega)$ equipped with the norm

$$
u \mapsto\|u\|_{L^{p(.)}(\Omega)}+\|\nabla u\|_{L^{p(\cdot)}(\Omega)}
$$

becomes a Banach space.

If (4) is satisfied, then, $W_{0}^{1, p(.)}(\Omega)$ is the closure of the set $\mathscr{C}_{0}^{\infty}(\Omega)$ with respect to the norm of $W^{1, p(.)}(\Omega)$.

If the boundary $\partial \Omega$ of $\Omega$ is Lipschitz and $p \in \mathscr{C}^{0}(\Omega)$ the norm of $W_{0}^{1, p(.)}(\Omega)$ is equivalent to the following norm

$$
u \mapsto\|\nabla u\|_{L^{p(.)}(\Omega)}
$$

(6) Friedrich's inequality is valid in the following form: if $p($.$) satisfies conditions (2), (3), and (4), then there$ exists constants $C>0$ such that

$$
\|u\|_{L^{p(\cdot)}(\Omega)} \leq C\|\nabla u\|_{L^{p(\cdot)}(\Omega)} \cdot
$$

(7) Under (2), $W^{1, p(.)}(\Omega)$ is separable and reflexive

\section{B. Epiconvergence Notion}

In this subsection, we will give a notion of operator's sequence convergence, named epiconvergence, who is a special case of the $\Gamma$-convergence introduced by De Giorgi and Spagnolo (1979) [19]. It is well suited to the asymptotic analysis of sequences of minimization problems.

Definition B.1 (see [18], Definition 1.9). Let $(\mathbb{X}, \tau)$ be a metric space and $\left(F^{\varepsilon}\right)_{\varepsilon}$ and $F$ be functionals defined on $\mathbb{X}$ and with value in $\mathbb{R} \cup\{+\infty\}$. $F^{\varepsilon}$ epiconverges to $F$ in $(\mathbb{X}, \tau)$, noted $\tau-\underset{\varepsilon \longrightarrow 0}{\operatorname{epilim}} F^{\varepsilon}=F$, if the following assertions are satisfied

(1) For all $x \in \mathbb{X}$, there exists $x_{\varepsilon}^{0}, x_{\varepsilon}^{0} \longrightarrow^{\tau} x$ such that $\lim \sup _{\varepsilon \longrightarrow 0} F^{\varepsilon}\left(x_{\varepsilon}^{0}\right) \leq F(x)$

(2) For all $x \in \mathbb{X}$ and all $x_{\varepsilon}$ with $x_{\varepsilon} \longrightarrow{ }^{\tau} x$, $\lim _{\text {inf }} \longrightarrow 0 F^{\varepsilon}$ $\left(x_{\varepsilon}\right) \geq F(x)$

Note the following stability result of the epiconvergence.

Proposition B.2 (see [18], p. 40). Suppose that $F^{\varepsilon}$ epiconverges to $F$ in $(\mathbb{X}, \tau)$ and that $\Phi: \mathbb{X} \longrightarrow \mathbb{R} \cup\{+\infty\}$ is $\tau$ -continuous. Then, $F^{\mathcal{E}}+\Phi$ epiconverges to $F+\Phi$ in $(\mathbb{X}, \tau)$.

Theorem B.3 (see [18], Theorem 1.10). Suppose that

(1) $F^{\varepsilon}$ admits a minimizer on $\mathbb{X}$

(2) The sequence $\left(\bar{u}^{\varepsilon}\right)$ is $\tau$-relatively compact

(3) The sequence $F^{\varepsilon}$ epiconverges to $F$ in this topology $\tau$

Then, every cluster point $\bar{u}$ of the sequence $\left(\bar{u}^{\varepsilon}\right)$ minimizes F on $\mathbb{X}$ and

$$
\lim _{\varepsilon^{\prime} \longrightarrow 0} F^{\varepsilon^{\prime}}\left(\bar{u}^{\varepsilon^{\prime}}\right)=F(\bar{u})
$$

if $\left(\bar{u}^{\varepsilon^{\prime}}\right)_{\varepsilon}{ }^{\prime}$ denotes the subsequence of $\left(\bar{u}^{\varepsilon}\right)_{\varepsilon}$ which converges to $\bar{u}$

Lemma B.4 (see [15], Appendix). Let $\varphi \in L^{\infty}(\Sigma)$, a Y-periodic, $Y=] 0,1[\times] 0,1[$. Let

$$
\varphi_{\varepsilon}(x)=\varphi\left(\frac{x}{\varepsilon}\right), \text { for a small enough } \varepsilon>0 \text {. }
$$

So that

$$
\begin{gathered}
\varphi_{\varepsilon} \longrightarrow m(\varphi) \text { in } L^{s}(\Sigma) \text { for } \quad 1 \leq s<\infty \\
\varphi_{\varepsilon} \rightarrow^{*} m(\varphi) \text { in } L^{\infty}(\Sigma) .
\end{gathered}
$$

\section{Data Availability}

No data were used to support this study.

\section{Conflicts of Interest}

The authors declare that they have no conflicts of interest.

\section{Acknowledgments}

The authors would like to thank the referee(s) for his/her comments and suggestions on the manuscript.

\section{References}

[1] A. A. Koshelev, A. E. Bazhutina, S. F. Pravdin, K. S. Ushenin, L. B. Katsnelson, and O. E. Solovyova, "A modified 
mathematical model of the anatomy of the cardiac left ventricle," Complex Systems Biophysics, vol. 61, no. 5, pp. 785-792, 2016.

[2] M. Ružíčka, "Electrorheological Fluids: Modeling and Mathematical Theory," in Lecture Notes in Math, vol. 1748, Springer, Berlin, 2000.

[3] A. A. Moussa and C. Licht, "Comportement asymptotique d'une plaque mince non linéaire," J. Math du Maroc, no. 2, pp. 1-16, 1994.

[4] A. Bourgeat and R. Tapiero, "Homogénéisation d'une plaque mince, thermoélastique, perforée transversalement de structure non uniformément périodique," Comptes rendus des séances de l'Académie des sciences. Série 1, Mathématique, vol. 297, no. 3, pp. 213-216, 1983.

[5] P. Suquet, A. Sawczuck, and G. Bianchi, Eds.G. Bianchi and A. Sawczuk, "Plasticity Today: Modelling," in Methods and Applications, P. Suquet, A. Sawczuck, and G. Bianchi, Eds., pp. 279-310, Elsevier Applied Science Publishers, 1985.

[6] A. Brillard, "Asymptotic analysis of nonlinear thin layers," International Series of Numerical Mathematics, vol. 123, pp. 321-338, 1997.

[7] J. F. Ganghoffer, A. Brillard, and J. Schultz, "Modelling of mechanical behavior of joints bounded by a nonlinear incompressible elastic adhesive," European Journal of Mechanics. A/Solids, vol. 16, pp. 255-276, 1997.

[8] J. M. Arrieta and M. Villanueva-Pesqueira, "Thin domains with non-smooth periodic oscillatory boundaries," Journal of Mathematical Analysis and Applications, vol. 446, no. 1, pp. 130-164, 2017.

[9] G. Cardone, C. Perugia, and M. Villanueva Pesqueira, "Homogenization of Bingham Flow in a thin domain with an oscillating boundary," pp. 1-23, 2019, https://arxiv.org/ abs/1905.02126/.

[10] J. M. Arrieta, A. N. Carvalho, M. C. Pereira, and R. P. Silva, "Semilinear parabolic problems in thin domains with a highly oscillatory boundary," Nonlinear Analysis: Theory, Methods and Applications, vol. 74, no. 15, pp. 5111-5132, 2011.

[11] R. P. Silva, "Behavior of the -Laplacian on thin domains," International Journal of Differential Equations, vol. 2013, Article ID 210270, 4 pages, 2013.

[12] J. M. Arrieta and M. C. Pereira, "Homogenization in a thin domain with an oscillatory boundary," Journal de Mathématiques Pures et Appliquées, vol. 96, no. 1, pp. 29-57, 2011.

[13] M. G. D. Geers, V. G. Kouznetsova, and W. A. M. Brekelmans, "Multi-scale computational homogenization: trends and challenges," Journal of Computational and Applied Mathematics, vol. 234, no. 7, pp. 2175-2182, 2010.

[14] H. P. Huy and E. Sanchez-Palencia, "Phenomenes de transmission a travers des couches minces de conductiviteelevee," Journal of Mathematical Analysis and Applications, vol. 47, no. 2, pp. 284-309, 1974.

[15] A. Ait Moussa and J. Messaho, "Limit behavior of an oscillating thin layer," in Electronic Journal of Differential Equations, Conference, vol. 14, pp. 21-33, Oujda International Conference on Nonlinear Analysis, Oujda, Morocco, 2006.

[16] J. Messaho and K. Hilal, "Limit behavior of a heat loss problem on oscillating thin layer," Boletim da Sociedade Paranaense de Matematica, vol. 35, no. 1, pp. 147-163, 2017.

[17] B. Amaziane, S. Antontsev, L. Pankratov, and A. Piatnitski, "Homogenization of $p$-Laplacian in perforated domain,"
Annales de l'Institut Henri Poincaré C, Analyse non linéaire, vol. 26, no. 6, pp. 2457-2479, 2009.

[18] H. Attouch, Variational Convergence for Functions and Operators, Pitman, London, 1984.

[19] E. De Giorgi and S. Spagnolo, "Convergence problems for functions and operators proceedings of the international," in Meeting on $\backslash$ Recent Methods in Nonlinear Analysis, E. Giorgi, E. Magenes, and U. Mosco, Eds., pp. 131-188, Pitagora, Rome Bologna, 1979.

[20] H. Brezis, Analyse fonctionnelle, Théorie et Applications, Masson, 1992.

[21] X. Fan and Q. Zhang, "Existence of solutions for $p(x)$-Laplacian Dirichlet problem," Nonlinear Analysis: Theory, Methods \& Applications, vol. 52, no. 8, pp. 1843-1852, 2003.

[22] X. Fan, "Boundary trace embedding theorems for variable exponent Sobolev spaces," Journal of Mathematical Analysis and Applications, vol. 339, no. 2, pp. 1395-1412, 2008.

[23] O. Kováčik and J. Rákosník, "On spaces $\mathrm{L}^{\mathrm{p}(\mathrm{x})}$ and $\mathrm{W}^{\mathrm{k}, \mathrm{p}(\mathrm{x})}$," Czechoslovak Mathematical Journal, vol. 41, no. 4, pp. 592618, 1991.

[24] L. Diening, P. Harjulehto, P. Hästö, and M. Ružíčka, "Lebesgue and Sobolev Spaces with Variable Exponents," in Lecture Notes in Mathematics, vol. 2017, Springer, Heidelberg, 2011. 